CERN-PH-TH/2007-184

ITP-UH-22/07

\title{
Noncommutative Solitons
}

\author{
Olaf Lechtenfeld \\ Theory Division, Physics Department, CERN, CH-1211 Geneva 23, Switzerland \\ on leave from: Institut für Theoretische Physik, Leibniz Universität Hannover
}

\begin{abstract}
Solitonic objects play a central role in gauge and string theory (as, e.g., monopoles, black holes, D-branes, etc.). Certain string backgrounds produce a noncommutative deformation of the low-energy effective field theory, which allows for new types of solitonic solutions. I present the construction, moduli spaces and dynamics of Moyal-deformed solitons, exemplified in the $2+1$ dimensional Yang-Mills-Higgs theory and its Bogomolny system, which is gauge-fixed to an integrable chiral sigma model (the Ward model). Noncommutative solitons for various $1+1$ dimensional integrable systems (such as sine-Gordon) easily follow by dimensional and algebraic reduction. Supersymmetric extensions exist as well and are related to twistor string theory.
\end{abstract}

Keywords: solitons, noncommutative field theory, integrable systems

PACS: $05.45 . \mathrm{Yv}, 11.10 . \mathrm{Nx}, 02.30 . \mathrm{Ik}$

\section{BEATING DERRICK'S THEOREM}

\subsection{Solitons in $d=1+2$ scalar field theory}

The simplest relativistic field theory in flat three-dimensional spacetime deals with a real scalar field $\phi$ depending on time $t$ and spatial coordinates $z=x+\mathrm{i} y$, governed by an action

$$
S^{(0)}[\phi]=\quad \mathrm{d} t \mathrm{~d} x \mathrm{~d} y \quad \frac{1}{2} \dot{\phi}^{2} \quad \frac{1}{2}(\nabla \phi)^{2} \quad V(\phi) \quad ;
$$

where I take the potential to be polynomial and bounded by zero from below, i.e.

$$
V^{0}(\phi)=v \prod_{i}\left(\begin{array}{ll}
\phi & \phi_{i}
\end{array}\right) \quad \text { and } \quad V(\phi) \quad 0 \quad \text { with } \quad V\left(\phi_{0}\right)=0 ;
$$

so that $\phi_{i}$ denote the (constant) extrema of $V$, including the vacuum $\phi_{0}$. Static classical field configurations $\varnothing$ extemize the energy

$$
E^{(0)}[\phi]=\quad \mathrm{d} x \mathrm{~d} y \frac{1}{2}(\nabla \phi)^{2}+V(\phi) ;
$$

but Derrick's theorem rules out any interesting solutions: Given some extremum $\Phi(x ; y)$ of $E^{(0)}$, I may consider the family $\phi_{\lambda}(x ; y):=\varnothing\left(\frac{x}{\lambda} ; \frac{y}{\lambda}\right)$ of rescaled configurations, whose

${ }^{1}$ Talk given at the Third Mexican Meeting on Mathematical and Experimental Physics at El Colegio Nacional, Mexico City, 10-14 September 2007 
energy must be extremized by $\phi_{1}(x ; y)$. However, the $\lambda$-variation of

$$
E^{(0)}(\lambda):=E^{(0)}\left[\phi_{\lambda}\right]=\lambda^{{ }^{Z}} \mathrm{~d} x \mathrm{~d} y \frac{1}{2}(\nabla \phi)^{2}+\lambda^{2} \mathrm{~d} x \mathrm{~d} y V(\phi)
$$

at $\lambda=1$ only vanishes for $V(\varnothing) \quad 0$, meaning $\phi=\phi_{0}$. Thus, barring possible vacuum degeneracy, the only non-singular static solution to the equation of motion is the vacuum.

\subsection{Noncommutative deformation}

One possible way out is a noncommutative deformation of the $x y$ plane, the simplest of which is the Moyal deformation

$$
(x ; y) \quad ! \quad(X ; Y) \quad \text { with } \quad[X ; Y]=\mathrm{i} \theta=\text { const : }
$$

For definiteness, one may realize the noncommuting spatial coordinates $X$ and $Y$ by infinite-dimensional matrices

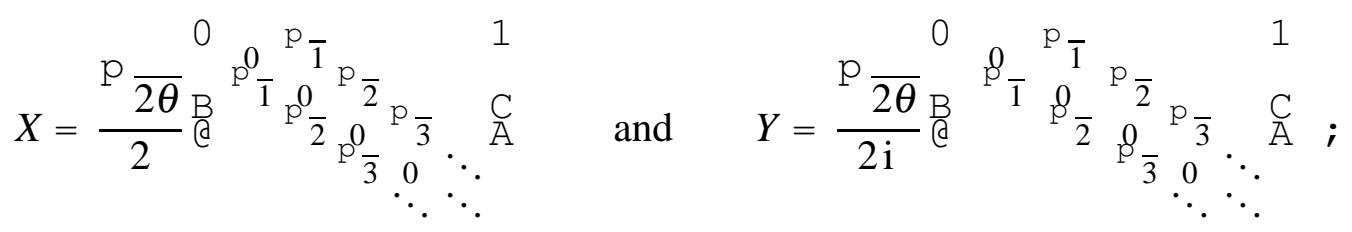

which act on some auxiliary (Fock) space $\mathscr{F}$. More generally, sufficiently nice functions $f(t ; x ; y)$ turn into bounded operators $F(t)$ via

$$
f(t ; x ; y) \quad ! \quad F(t)=\text { Weyl-order } f(t ; X ; Y) \quad \text { acting on } \mathscr{F} ;
$$

where every monomoial on the right-hand side is meant to be Weyl (or symmetrically) ordered in $X$ and $Y$. Furthermore, derivatives become inner derivations in the operator algebra, and the spatial integral translates to a trace over $\mathscr{F}$,

$$
\partial_{x} ! \quad \frac{\mathrm{i}}{\theta}[Y ; \quad] ; ! \quad \frac{\mathrm{i}}{\theta}[X ; \quad] \quad \text { and } \quad \mathrm{d} x \mathrm{~d} y f=\pi \theta \operatorname{tr}_{\mathscr{F}} F \text { : }
$$

I try to use corresponding small and capital letters for functions and operators, respectively. The energy on the Moyal plane takes the form

$$
E^{(\theta)}=\pi \operatorname{tr} \quad \frac{1}{2}\left[p_{\bar{\theta}}^{X} ; \Phi\right]^{2} \quad \frac{1}{2}\left[\frac{Y}{\bar{\theta}} ; \Phi\right]^{2}+\theta V(\Phi) \quad \theta ! !^{\infty} \quad \pi \theta \operatorname{tr} V(\Phi) ;
$$

which in the large- $\theta$ limit is stationary for operators $\Phi$ subject to

$$
0=V^{0}(\Phi) \quad v\left(\Phi \phi_{0}\right)\left(\Phi \phi_{1}\right) \quad \Phi\left(\phi_{n}\right):
$$




\subsection{New classical solutions}

Because of its operator-valuedness, Derrick's theorem is avoided, and (10) admits a large set of non-constant solutions [1],

$$
\Phi=\sum_{i} \phi_{i} P_{i} \quad \text { with } \quad P_{i} P_{j}=\delta_{i j} P_{j} \quad \text { and } \quad \sum_{i} P_{i}=\mathbb{1} ;
$$

based on a partition of the identity into orthogonal projectors $P_{i}$ associated to the extrema $\phi_{i}$, allowing for zero projectors. This is easily checked,

$$
\begin{aligned}
& \prod_{k}\left(\begin{array}{ll}
\Phi & \phi_{k}
\end{array}\right)=\prod_{k}\left(\sum_{i} \phi_{i} P_{i} \quad \phi_{k} \mathbb{1}\right)=\prod_{k} \sum_{i}\left(\begin{array}{ll}
\phi_{i} & \phi_{k}
\end{array}\right) P_{i} \\
& =\sum_{i_{1} ;:: i_{n}} \prod_{k}\left(\phi_{i_{k}} \quad \phi_{k}\right) P_{i_{k}}=\sum_{i} P_{i} \prod_{k}\left(\begin{array}{ll}
\phi_{i} & \phi_{k}
\end{array}\right)=0:
\end{aligned}
$$

Hence,

$$
E^{(\theta)}[\Phi] \quad \theta ! !^{\infty} \quad \pi \theta \sum_{i} V\left(\phi_{i}\right) \operatorname{tr} P_{i}=: \theta E_{0}
$$

is degenerate over the infinite-dimensional moduli space $\frac{{ }^{N} \mathrm{U}(\mathscr{F})}{i \mathrm{U}\left(\operatorname{rank} P_{i}\right)}$ at $\theta=\infty$. At a finite value of $\theta$ I should expand around $\theta=\infty[2,3]$,

$$
\begin{aligned}
& E_{(\theta)}\left[\Phi_{\mathrm{cl}}\right]=\theta E_{0}+E_{1}+\frac{1}{\theta} E_{2}+::: \text { for } \Phi_{\mathrm{cl}}=\Phi+\frac{1}{\theta} \Phi^{0}+::: ; \\
& \text { with } \quad E_{1}=\frac{\pi}{2} \sum_{i ; j} \phi_{i} \phi_{j} \operatorname{tr}\left[\frac{X}{\bar{\theta}} ; P_{i}\right]\left[\frac{X}{\bar{\theta}} ; P_{j}\right]+\left[\frac{Y}{\bar{\theta}} ; P_{i}\right]\left[\frac{Y}{\bar{\theta}} ; P_{j}\right]:
\end{aligned}
$$

\subsection{Double-well example}

I illustrate the above with a double-well potential

$$
V(\phi)=\beta\left(\begin{array}{lll}
\phi^{2} & 1
\end{array}\right)^{2} \quad ! \quad V^{0}(\phi)=4 \beta(\phi+1) \phi(\phi \quad 1)
$$

and link projectors $P\left(\phi_{i}\right)$ to the three extrema $\phi_{i}=1 ; 0 ;+1$,

$$
P(1)=: P ; \quad P(0)=\mathbf{0} ; \quad P(+1)=\mathbb{1} P: \quad \text { Hence, }
$$

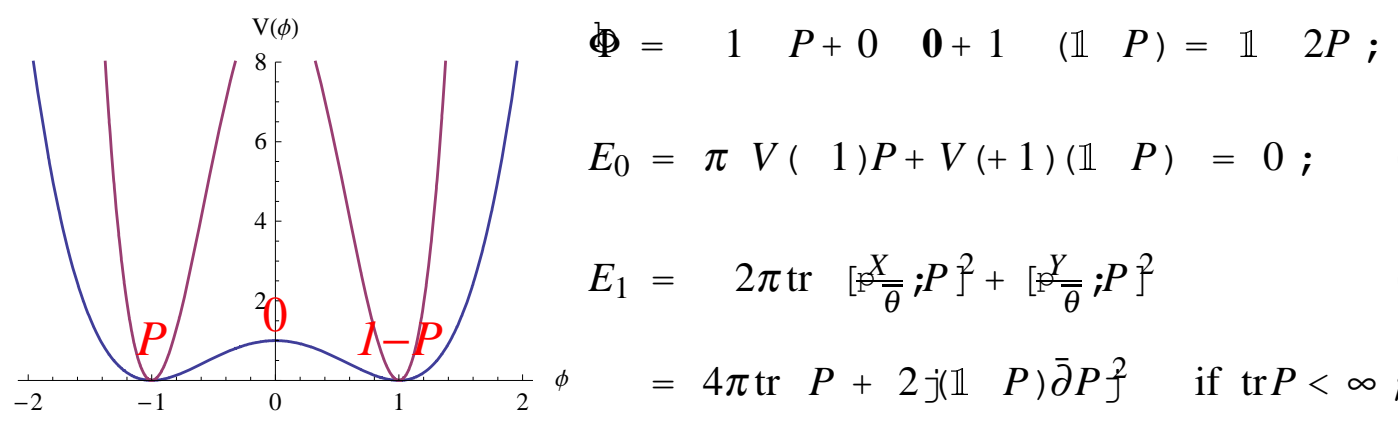


where I introduced the abbreviation

$$
\left.\bar{\partial}:=\left[\frac{X+\mathrm{i} Y}{\overline{2 \theta}} ; \quad\right]=\frac{Z}{\overline{2 \theta}} ; \quad\right]=:[a ; \quad]
$$

in the last line. Clearly, BPS saturation $E_{1}=4 \pi \operatorname{tr} P$ is reached for $\bar{\partial}$-stable projectors, i.e. when $a: \operatorname{im} P ! \operatorname{im} P$. It is easy to see that all further corrections $\phi^{0}, E_{2}$, etc. are of $O(1=\beta)$, whence $\Phi$ and $E_{1}$ become exact for $\beta$ ! $\infty[4]$.

\section{SIGMA MODEL SOLITONS}

\subsection{Deformed sigma models}

The double-well example is directly related to a sigma model, since the $\beta ! \infty$ limit nails $\phi$ to the vacuum 'manifold' $f 1 ;+1 \mathrm{~g}$. For a more interesting situation, let me take $\phi$ to be complex valued and change the double-well to a Mexican-hat potential, so that

$$
\beta ! \infty \quad ! \quad \Phi \Phi^{\dagger}=\mathbb{1} ;
$$

i.e. we have a $\mathrm{U}(1)_{\theta}$ sigma model. $^{2}$ The nonabelian generalization to $\Phi 2 \mathrm{U}(n)_{\theta}$ is obvious; I just have to perform the traces over both $\mathrm{C}^{n}$ and $\mathscr{F}$, as for example in

$$
E^{(\theta)}=2 \pi \operatorname{tr} \partial \Phi^{\dagger} \bar{\partial} \Phi=2 \pi \operatorname{tr}[a ; \Phi]^{2} \quad \text { with } \quad\left[a ; a^{\dagger}\right]=\mathbb{1} ;
$$

where the Heisenberg algebra follows from $[Z ; \bar{Z}]=2 \theta \mathbb{1}$. The configuration space $\mathrm{U}(n)_{\theta}$ contains the important Grassmannian subsectors

$$
\mathrm{Gr}(k ; \infty)=\frac{\mathrm{U}(n)_{\theta}}{\mathrm{U}(k) \mathrm{U}(\infty \quad k)}=\frac{\mathrm{U}\left(\mathrm{C}^{n} \mathscr{F}\right)}{\mathrm{U}(\operatorname{im} P) \mathrm{U}(\operatorname{ker} P)} \quad \text { for } \quad k=\operatorname{tr} P ;
$$

which are the spaces of hermitian $\Phi$ based on hermitian rank- $k$ projectors,

$$
\Phi^{\dagger}=\Phi \quad \$ \quad \Phi^{2}=\mathbb{1} \quad \$ \quad \frac{1}{2}(\mathbb{1} \quad \Phi)=P=P^{2} \quad \$ \quad \Phi=\mathbb{1} \quad 2 P:
$$

In each Grassmannian I can define a topological charge

$$
Q=\frac{1}{8} \operatorname{tr} \Phi[\partial \Phi ; \bar{\partial} \Phi] \quad(=\operatorname{tr} P \quad \text { if } \operatorname{tr} P \text { is finite }) ;
$$

and the energy has a lower BPS bound [5]

$$
E_{\mathrm{BPS}}=8 \pi Q j \quad \text { attained for } \quad(\mathbb{1} \quad P) \bar{\partial} P=0:
$$

Adding a constant to $\Phi$ does not change $Q$, but interchanging $P$ with $\mathbb{1} \quad P$ flips its sign.

2 The $\theta$ subscript means that the U(1)-valued $\phi$ lives on the Moyal plane or that $\Phi$ acts on $\mathrm{C} \mathscr{F}$. 


\subsection{Noncommutative solitons}

In the commutative $\mathrm{U}(n)$ sigma model for $n>1$, the BPS configurations

$$
\phi=\mathbb{1} \quad 2 P \quad \text { with } \quad(\mathbb{1} \quad P) \bar{\partial} P=0
$$

are static solitons, provided the projector $P(x ; y)$ is a polynomial $n \quad n$ matrix function of $x$ and $y$. A constantly moving single-soliton configuration is generated by a Lorentz boost,

$$
P(x ; y) \eta \quad P \frac{x v_{x} t}{1 v^{2}} ; \frac{y v_{y} t}{1 v^{2}} \quad \text { and } \quad E \geqslant \frac{1}{1 v^{2}} E:
$$

The same is true for the Moyal-deformed theory (via $x ! X$ and $y ! Y$ ), but here arises the novel possibility of abelian solitons as I will outline [6, 7]. In any case, these solitons extremize the action

$$
S_{2}=\frac{1}{2}^{\mathrm{z}} \mathrm{d} t \pi \theta \operatorname{tr} \eta^{\mu v} \partial_{\mu} \Phi^{\dagger} \partial_{\nu} \Phi=\frac{\pi \theta}{2}_{t}^{\mathrm{z}} \operatorname{tr} A^{\wedge} \quad A \quad \text { with } \quad A:=\Phi^{\dagger} \mathrm{d} \Phi
$$

being an auxiliary flat connection. In a non-integrable model, we cannot expect exact multi-soliton solutions. Indeed, numerical studies in the commutative case show that scattered sigma-model lumps are unstable: they radiate and shrink, but may live long enough to repel each other and even scatter head-on at 90 . I can, however, pass to an integrable model (featuring exact multi-solitons) by adding a WZW-like term $S_{3}$ to the action. I will consider the resulting so-called Ward model [8] from now on.

\section{WARD MODEL SOLITONS}

\subsection{U(n) Ward model}

I begin with the undeformed situation and add to $S_{2}$ the WZW-like term [8]

$$
S_{3}=\frac{1}{3}_{0}^{\mathrm{Z}_{1} \mathrm{Z}} \mathrm{R}^{2 ; 1} V^{\wedge} \operatorname{tr} \AA^{\wedge} \mathbb{A} \AA \quad \text { with an extension } \quad \mathbb{A}\left(\lambda={ }_{0}^{1}\right)={ }_{0}^{A}
$$

and a Lorentz-breaking one-form $V=\mathrm{d} x$. The equation of motion then changes to

$$
0=\left(\eta^{\mu v}+V_{\rho} \varepsilon^{\rho \mu v}\right) \partial_{\mu}\left(\phi^{\dagger} \partial_{v} \phi\right)=\partial_{x}\left(\phi^{\dagger} \partial_{x} \phi\right)+\partial_{y t}\left(\phi^{\dagger} \partial_{y+t} \phi\right)
$$

and remains boost invariant only in the $y$ direction. Static solitons do not see $S_{3}$ and, hence, still have the form

$$
\phi=\mathbb{1} \quad 2 P \quad \text { with the BPS condition } \quad \bar{\partial} P=P \Gamma ;
$$

where $\Gamma(x ; y)$ is an arbitrary $n \quad n$ matrix function. Due to the reduced Lorentz invariance, however, their boosted cousins may suffer a shape change [8],

$$
\phi=(\mathbb{1} \quad P)+\frac{\mu}{\bar{\mu}} P=\mathbb{1} \quad\left(1 \quad \frac{\mu}{\bar{\mu}}\right) P ; \text { with } \quad \mu=\frac{\mathrm{v}_{x}+\mathrm{i}^{\mathrm{p}} \overline{1 y^{2}}}{1 \mathrm{v}_{y}} 2 \mathrm{CnR}
$$




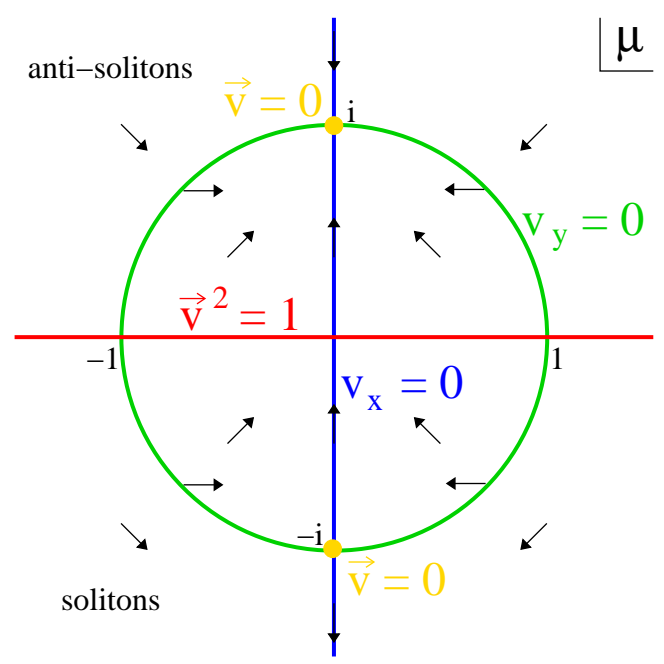

FIGURE 1. Velocity $\vee$ as function of rapidity $\mu$

serving as a rapidity parameter, and with a boosted argument of $P$. For $\mathrm{v}_{x}=0$ I note that i $\mu$ becomes the proper Lorentz contraction factor, and $\phi=\mathbb{1} \quad 2 P$ again. Clearly, $\mu=0$ is the static case. Each polynomial BPS projector $P$ then yields a soliton with velocity $\left(\mathrm{v}_{x} ; \mathrm{v}_{y}\right)$ and energy $E$ given by (see Figure 1) [8]

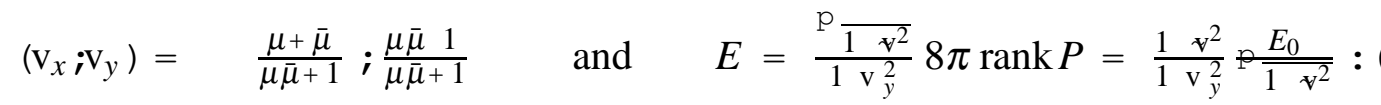

\subsection{Relation with $2+2$ self-dual Yang-Mills and $2+1$ Bogomolny}

Being integrable, the Ward sigma model should descend from self-dual Yang-Mills in $2+2$ dimensions via the Bogomolny system in $2+1$ dimensions [8]. Their first-order field equations imply the second-order full Yang-Mills and Yang-Mills-Higgs equations, respectively:

$$
\begin{array}{cccc}
d=2+2: & D^{m} f_{m n}=0 & (= & f_{m n}=\frac{1}{2} \varepsilon_{m n p q} f^{p q} \\
\# & \# & \# \\
d=2+1: & D^{\mu} f_{\mu v}=h D_{v} h & (= & f_{\mu v}=\varepsilon_{\mu v \rho} D^{\rho} h
\end{array} ; ;
$$

with obvious notation. A light-cone ansatz [9],

$$
\begin{aligned}
& a_{t y}=0 \quad \text { and } \quad a_{x} \quad h=0 \text {; } \\
& a_{t+y}=\phi^{1} \partial_{t+y} \phi \quad \text { and } \quad a_{x}+h=\phi^{1} \partial_{x} \phi
\end{aligned}
$$

with $\phi(t ; x ; y) 2 \mathrm{U}(\mathrm{n})$, solves two of the three Bogomolny equations. The remaining equation precisely yields the (second-order) "Yang equation" (33) for Ward's sigma model,

$$
\partial_{x}\left(\phi^{\dagger} \partial_{x} \phi\right)+\partial_{y t}\left(\phi^{\dagger} \partial_{y+t} \phi\right)=0:
$$

Therefore, (28) is a "second-stage" BPS bound. 


\subsection{Ward multi-solitons}

The Ward model features a Lax pair and a linear system, which may be employed to construct exact nonabelian commutative multi-soliton configurations. The analysis reveals two types of solutions: First [6], generically one finds no-scattering configurations $\phi=\prod_{k} \mathbb{1} \quad\left(1 \quad \frac{\mu_{k}}{\bar{\mu}_{k}} P_{k} \quad\right.$ based on a collection $f\left(P_{k} ; \mu_{k}\right) g_{k=1:: m}$ of (non-orthogonal) individually boosted projectors. Their energy densities show $m$ lumps moving about the $x y$ plane with constant velocities $\forall_{k=1}:: m$ and oblivious to each other. Second [7], in asymptotically-coinciding-velocity limits $v_{k} v, ! 0$ for $t ! \infty$, novel scattering configurations emerge. For instance, two lumps ' $k$ ' and ' ", without relative motion at infinity, accelerate toward each other and scatter (at rational angles when head-on). Alternatively, one finds ring-like time-dependent multi-soliton bound states. In the noncommutatively deformed Ward model, such configurations occur even in the abelian case, i.e. for $\mathrm{U}(1)_{\theta}$. To understand these, I must characterize the corresponding projectors.

\subsection{Projectors of infinite and finite rank}

Recall that soliton configurations are constructed from hermitian projectors $P$. In the undeformed $\mathrm{U}\left(\begin{array}{ll}n & 2\end{array}\right)$ (Ward) sigma model, $P$ simply acts on the "color space" $\mathrm{C}^{n}$ and has "color rank" $r_{c}$. In the deformed $\mathrm{U}(n-1)_{\theta}$ (Ward) sigma model, however, $P$ acts on the product space $\mathrm{C}^{n} \mathscr{F} \mathscr{F}^{\prime} \quad \mathscr{F}$, with a "total rank" $r_{\mathrm{F}+r}{ }_{n^{+}} \mathrm{in}$ a color-diagonal basis. The obvious situation is a smooth deformation $P \quad \operatorname{l} P\left(X_{;} ;\right)$ of a commutative diagonal color-rank- $r_{c}$ projector to an operator-valued diagonal $n \quad n$ matrix, with a total rank $r=\infty_{1}+:::+\infty_{r_{c}}+0+:::+0=r_{c} \quad \infty$. Yet, this is a very special case: In any block ' $i$ ' I could take $0<r_{i}<\infty$ and have $r \Leftrightarrow r_{c} \quad \infty$, impeding a smooth $\theta ! 0$ limit (since $r_{c}$ is ill-defined) [10]. The epitome of such a genuinely noncommutative configuration has $r_{i}<\infty \quad 8 i$, so that the total rank $r$ is finite. In particular, I may now choose $n=1$ and $P$ of finite rank $r$ inside $\mathscr{F}$. These "abelian projectors" are the most simple and also the most extremely noncommutative ones, and I will concentrate on them for the rest of this talk.

\section{ABELIAN MULTI-SOLITONS}

\subsection{Static abelian solitons}

How do the static $\mathrm{U}(1)_{\theta}$ solitons look like? I know that these are Grassmannian BPS configurations in $\mathrm{U}(\mathscr{F})$, i.e. [5]

$$
\Phi=\mathbb{1} 2 P \quad \text { with } \quad(\mathbb{1} \quad P) a P=0 \text { : }
$$

Any rank- $r$ hermitian projector in $\mathscr{F}$ decomposes via

$$
P=T T i \frac{1}{h T T i} \mathrm{~h} T j \quad \text { with } \quad T i \mathrm{i}=T_{1} i_{;} T_{2} i_{;}::: ; T_{r} i \quad:
$$


The collection of ket states $\mathcal{T}_{i}$ i $2 \mathscr{F}$ (arranged in a row) spans $\operatorname{im} P$ but is otherwise arbitrary. The BPS condition above then translates to $T$ i as

$$
a \operatorname{Ti}=\operatorname{Ti} A \quad \text { for some } r \quad r \text { matrix } A \text {; }
$$

i.e. I am looking for $a$-stable subspaces of dimension $r$. Generically I can diagonalize

$$
A \quad ! \quad \operatorname{diag}\left(\alpha_{1} ; \alpha_{2} ;:: ; ; \alpha_{r}\right)
$$

by a basis change inside $\operatorname{im} P .{ }^{3}$ Hence, the $T_{i}$ i are eigenkets of $a$, also known as coherent states $[1,6]$,

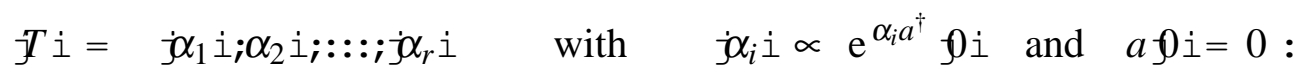

The corresponding BPS projector reads

$$
P_{r}=\sum_{i ; j=1}^{r} j \alpha_{i} \text { i h } \alpha_{:} \dot{j} \alpha_{: i}{ }_{i j}^{1} h \alpha_{j j} ;
$$

where the matrix of coherent-state overlaps must be inverted. For illustration I display the rank-one and rank-two cases (denoting $j i=a^{\dagger} 0 i$ ),

$$
\begin{aligned}
& P_{1}=\frac{j \alpha i h \alpha j}{h \alpha j \alpha \dot{i}}=\mathrm{e}^{j \alpha j^{2}} \mathrm{e}^{\alpha a^{\dagger}} \quad 0 \mathrm{ir} 0 j \mathrm{e}^{\bar{\alpha} a} \quad \alpha ! !^{0} \quad \oint i \nvdash 0 j ;
\end{aligned}
$$

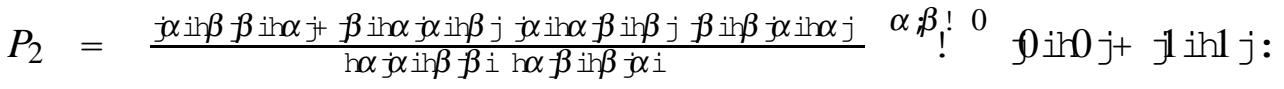

\subsection{Noncommutativity in function space: Moyal star product}

In order to develop some intuition for the properties of the noncommutative solitons and their deviation from the commutative ones, I may convert the Moyal-Weyl map (7) and associate my operators on $\mathscr{F}$ to ordinary functions on the $x y$ plane (with values in $\mathrm{U}(n))$. For this to be a homomorphism of the operator composition algebra, I must however deform the pointwise function product to the Moyal star product

$$
\begin{aligned}
& (f ? g)(x ; y)=f(x ; y) \exp \frac{\mathrm{i}}{2} \theta\left(\begin{array}{lll}
\partial_{x} \partial_{y} & \left.\partial_{y} \dot{\partial}_{x}\right) & g(x ; y)
\end{array}\right.
\end{aligned}
$$

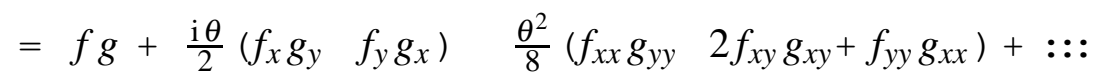

$$
\begin{aligned}
& =f g+\text { total derivatives : }
\end{aligned}
$$

The inverse of (7) then maps (usually not Weyl-ordered) operators $F=F(X ; Y)$ to functions $f_{\theta}=F_{?}(x ; y)$, where the star indicates the Moyal product in all compositions. The most important properties of the deformed product are

$$
(f ? g) ? h=f ?(g ? h) \quad ; \quad \operatorname{sd} x \mathrm{~d} y f ? g=\operatorname{sd} x \mathrm{~d} y f g \quad ; \quad x ? y \quad y ? x=\mathrm{i} \theta ;(50)
$$

\footnotetext{
${ }^{3}$ The general case gives Jordan blocks which pose no problem [2].
} 


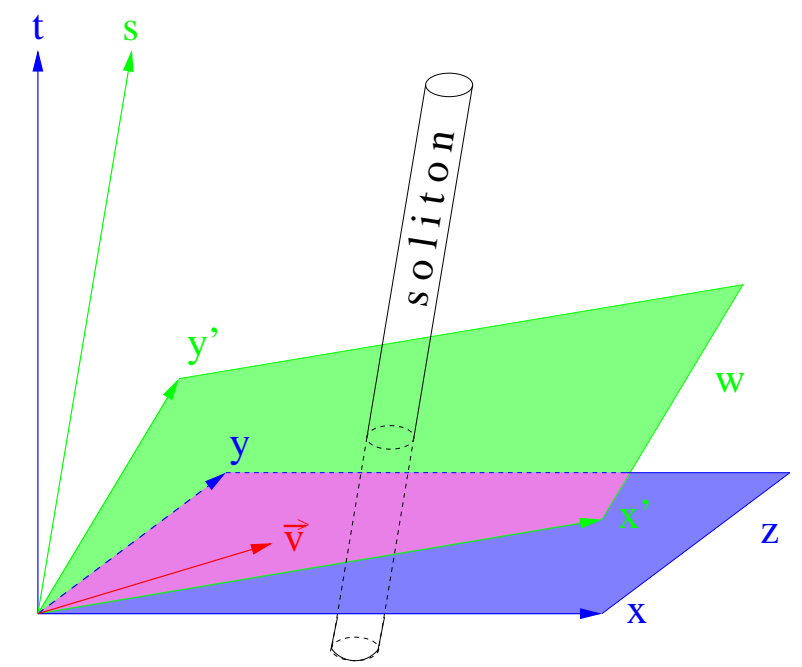

FIGURE 2. Soliton worldline and co-moving coordinates

and the key object for building the abelian solitons is the operator $\$$ function

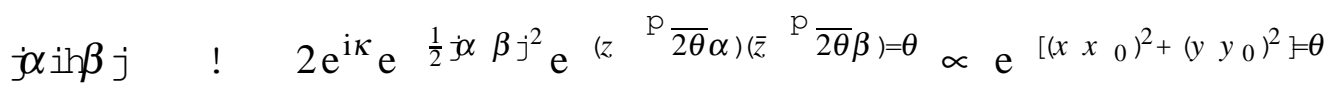

with constants $x_{0}, y_{0}$ and $\kappa$ depending on $\alpha$ and $\beta$. Clearly, the abelian soliton profile is Gaussian, as is its energy density. This representation also makes the $\operatorname{singular} \theta ! 0$ limit explicit.

\subsection{Abelian Ward solitons}

Let me now construct the moving solutions (35) for the abelian case. To formulate the BPS condition efficiently, I pass from the standard coordinates $(z ; \bar{z} ; t)$ to co-moving (or rest-frame) coordinates $(w ; \bar{w} ; s)$ in a linear way $[5,11]$ as depicted in Figure 2 . We also rescale $w$ such that the coordinate change is canonical, or preserves the noncommutativity relation (5) or (50), i.e. $[w ; \bar{w}] ?=2 \theta=[W ; \bar{W}]$ Normalizing

$$
W=\mathrm{P} \overline{2 \theta} c \quad \text { such that }\left[c ; c^{\dagger}\right]=\mathbb{1} ;
$$

$c$ is connected with $a$ by an ISU $(1,1)$ squeezing (or inhomogeneous Bogoliubov) transformation [6],

$$
c=S(t) a S(t)^{\dagger} \quad \text { and } \quad j \mathrm{j} i=S(t) 0 i \quad \text { with } \quad c j i=0 ;
$$

where I denote with $j i$ the co-moving vacuum ket. The BPS condition them simply reads

$$
\partial_{s} P=0 \quad \text { and } \quad(\mathbb{1} \quad P) c P=0 \quad ! \quad c j i=\mathbb{T} i A
$$


and is solved by $c$-coherent states (see (45)) [6]

$$
\mathcal{J}_{i} \mathrm{i} \propto \mathrm{e}^{\alpha_{i} c^{\dagger}} \dot{\mathrm{j} i}=S(t) j \alpha_{i} \dot{i}:
$$

It turns out that the soliton velocity and energy do not depend on $\theta$, but the $\mathrm{U}_{\text {? }}$ (1) soliton configuration is singular for $\theta$ ! 0 . The rank-one example reads

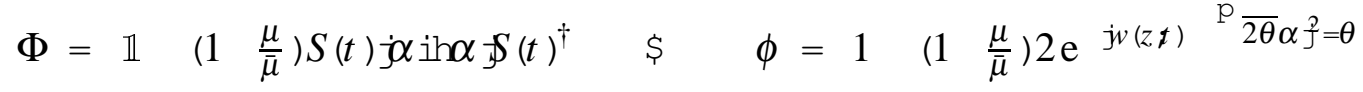

and represents a squeezed Gaussian lump moving with constant speed.

\subsection{Abelian Ward multi-solitons}

It is now clear how the construction of commutative Ward multi-solitons mentioned in subsection 3.3 carries over to the noncommutative situation. $\mathrm{A}_{\text {? }}$ (1) $m$-soliton configuration with rapidities $\left(\mu_{1} ;::: ; \mu_{m}\right)$ factorizes as

$$
\Phi=\prod_{k=m}^{1} \mathbb{1} \quad\left(1 \quad \frac{\mu}{\bar{\mu}}\right) P_{k} \quad \text { with } \quad P_{k}=\boldsymbol{j}^{(k)} \mathrm{i} \frac{1}{\mathrm{~h} T^{(k)} T^{(k)} \dot{\mathrm{i}}} \mathrm{h} T^{(k)} \mathrm{j}:
$$

The (rows of) kets $T^{(k)} i_{=} T_{i}^{(k)}{ }_{i=1 ;:: ; r_{k}}$ for $k=1 ;::: ; m$ can be found via [12]

$$
J_{i}^{(k)} i=\prod_{l=1}^{k 1} \mathbb{1} \quad \frac{\mu_{k l} \bar{\mu}_{k l} l}{\mu_{k} l \bar{\mu}_{k}} P_{k l} \quad S_{k}(t) \dot{\rho}_{i}^{k} \dot{i} ;
$$

where the factor in braces describes the background of lumps 1 to $k 1$ being felt by lump $k$ when it is added to the system. This allows for a recursive construction. As an illustration I present the simplest two-soliton example with $r_{1}=r_{2}=1[6,4]$ :

$$
\begin{aligned}
& \Phi^{\dagger}=\mathbb{1} \quad{\frac{1}{1 \mu j j^{2}}}^{\mathrm{n}} \frac{\mu_{11}}{\mu_{1}} j i h 1 j+\frac{\mu_{22}}{\mu_{2}} 2 i h 2 j \quad \sigma \mu \frac{\mu_{21}}{\mu_{2}} j i h 2 j \quad \bar{\sigma} \mu \frac{\mu_{12}}{\mu_{1}} 2 i h 1 j \\
& \text { with } \quad k i=S_{k}(t) \dot{\alpha}^{k} i ; \quad \sigma=h 12 i ; \quad \mu_{i j}=\mu_{i} \quad \bar{\mu}_{j} ; \quad \mu=\frac{\mu_{11} \mu_{22}}{\mu_{12} \mu_{21}} \text { : }
\end{aligned}
$$

\subsection{Abelian soliton scattering ?}

The static soliton moduli space $\mathrm{C}^{r}$ is parametrized by $\mathrm{f} \alpha_{i} g_{i=1 ; ;: ; r}$ which indicate the positions of the lumps in the Moyal plane. The bosonic nature of the lumps leads to identification under permutations, $\mathrm{C}^{r} ! \mathrm{C}^{r}=S_{r}$. I showed that generic abelian multisolitons are squeezed gaussian lumps, which move with mutually distict velocities $\mho_{k}$ and do not scatter. As in the commutative case, more interesting time dependencies arise in coinciding-velocity limits. The simplest case is the two-soliton solution (59), which for $\mu_{1 ; 2} ! \quad \mathrm{i}$ (static center of mass) tends to $[6,4]$

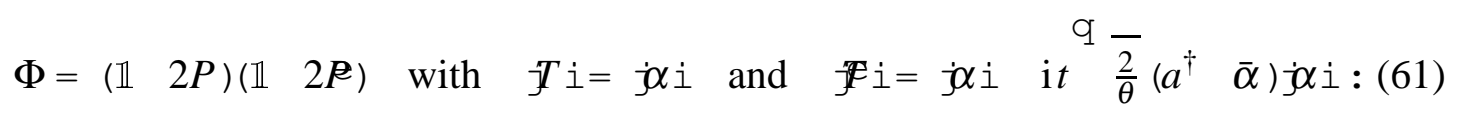



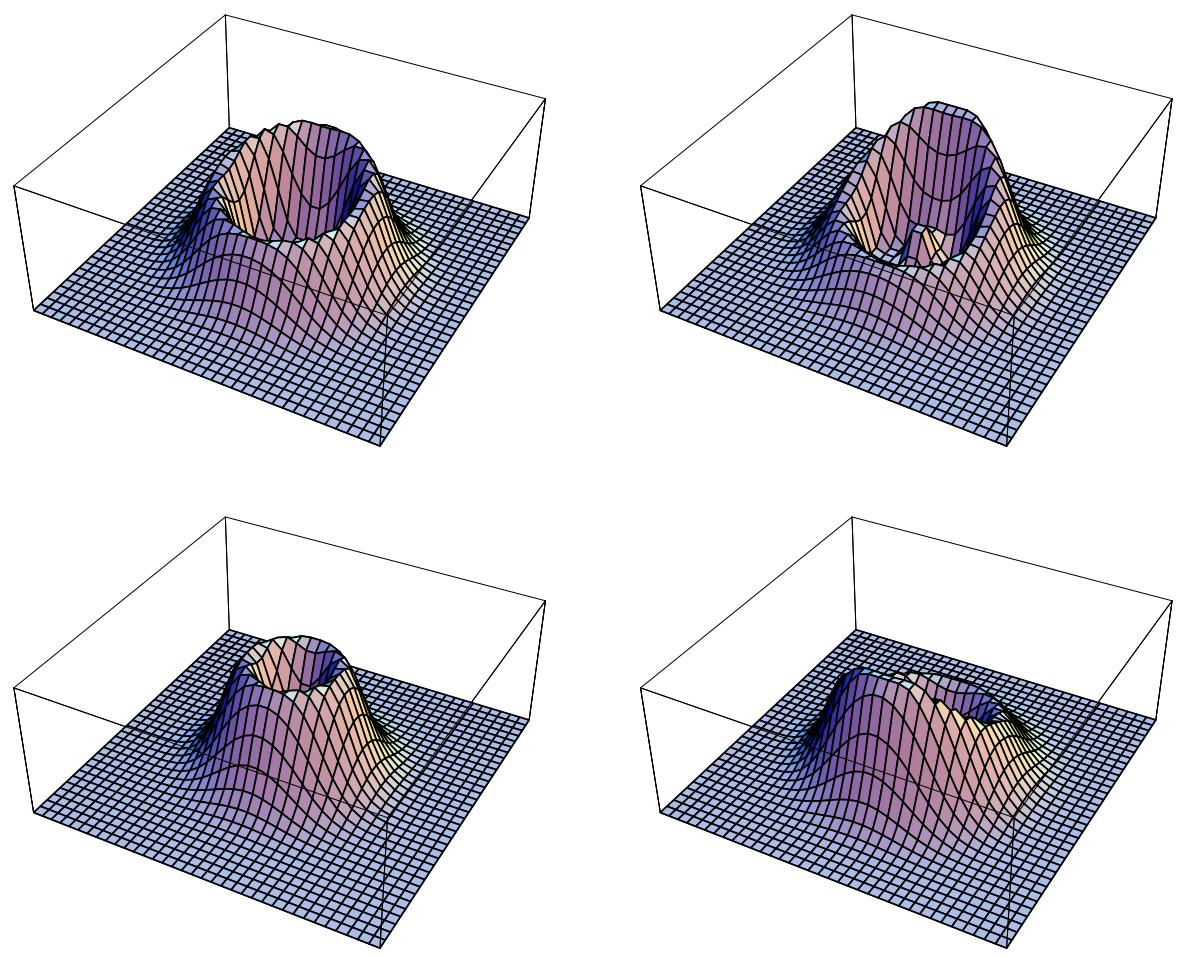

FIGURE 3. Energy density of abelian two-soliton at $\frac{t}{\bar{\theta}}=5 ; 15 ; 0 ; 15$

Taking $\alpha=0$ puts the center of mass at the origin and yields

$$
\phi=1 \quad{\frac{8 t}{\theta+2 t^{2}}}^{\mathrm{n}} 2 \frac{z \bar{z}}{\theta} t \quad \mathrm{i}(z+\bar{z}) \quad \mathrm{e}^{z \bar{z}=\theta} ;
$$

which is revealed not as a scattering configuration but as a ring-shaped bound state, which performs a single breath (see Figure 3). There do not seem to occur exact abelian Ward-model scattering solutions.

\subsection{Stability of static solitons}

An important issue is the stability of my solitons under small perturbations. The static solitons can be interpreted as solutions in the appropriate Grassmannian sigma model, where the BPS bound (28) guarantees their absolute stability. When these Grassmannians are imbedded into the general unitary sigma model, which must be considered for the time-dependent Ward solitons, topological stability disappears when stepping out of the Grassmannian. In fact, given any projector inclusion $P \quad P$ (admitting $P=0$ ), the path [10]

$$
\Phi(s)=\mathrm{e}^{\mathrm{i} s\left(\begin{array}{lll}
P & P
\end{array}\right)}(\mathbb{1} \quad 2 P)=\mathbb{1} \quad\left(1+\mathrm{e}^{\mathrm{i} s}\right) P \quad\left(\begin{array}{lll}
1 & \left.\mathrm{e}^{\mathrm{i} s}\right) P
\end{array}\right.
$$

connects $\Phi(0)=\Phi=\mathbb{1} \quad 2 P$ with $\Phi(\pi)=\Phi=\mathbb{1} \quad 2 P$ and thus interpolates between two static solitons in different Grassmannians inside $U(\mathscr{F})$. Without knowing any de- 
tails except for the topological charges $Q$ and $Q$, one can compute the energy [10]

$$
\frac{1}{8 \pi} E(s)=\frac{Q+Q}{2}+\frac{Q Q}{2} \cos s=Q \cos ^{2} \frac{s}{2}+Q \sin ^{2} \frac{s}{2} ;
$$

which decreases monotonically if $Q<Q$. Therefore, all Ward-model solitons decay to the "vacuum" $\Phi=\mathbb{1}$. For the diagonal abelian solitons, the instability subspace was shown to be one-dimensional, and the eigenmode was identified. In fact, from (36) I may compare the exact energy

$$
\begin{aligned}
\frac{1}{8 \pi} E_{\text {exact }} & =\frac{\theta}{8} \operatorname{tr} \dot{\Phi}(t \dot{\alpha})^{2}+\frac{1}{4} \operatorname{tr}[a ; \Phi(t j \alpha)]^{2}=\frac{1}{8 \pi}\left(E_{\text {kin }}+E_{\text {grad }}\right) \\
& =\frac{\mathrm{p} \overline{1 v^{2}}}{1 \mathrm{v}_{y}^{2}} \frac{1}{2} v^{2}+1 \frac{1}{2} v^{2} \quad \frac{1}{2}\left(\mathrm{v}_{x}^{2}+\mathrm{v}_{y}^{2}\right)+1 \mathrm{v}_{x}^{2}
\end{aligned}
$$

of the abelian one-soliton (56) at small speed to the adiabatic energy

$$
\frac{1}{8 \pi} E_{\text {adiab }}=\frac{\theta}{8} \operatorname{tr} \dot{\Phi}_{\text {static }}(\alpha(t))^{2}=\frac{1}{2}\left(\mathrm{v}_{x}^{2}+\mathrm{v}_{y}^{2}\right)+1
$$

in the moduli-space approximation $\Phi(t j \alpha) \quad \Phi_{\text {static }}(\alpha(t))$, after relating $\dot{\alpha}$ to v [4]. The difference is entirely due to the static deformation $2 P !\left(1 \frac{\mu}{\bar{\mu}}\right) P$ :

$$
\frac{1}{8 \pi} E_{\text {deform }}=\frac{1}{4} \operatorname{tr}[a ; \Phi(t j \alpha) \dot{\mathcal{S}}=1]^{2}=\frac{1 v^{2}}{1 \mathrm{v}_{y}^{2}} \quad 1 \quad \mathrm{v}_{x}^{2}:
$$

Therefore, these solitons loose energy by self-accelerating in the $x$ direction, i.e. the Lorentz violation is also the doom for their stability.

\section{NONCOMMUTATIVE SINE-GORDON}

\subsection{Dimensional and algebraic reduction}

The Ward model is a gateway to integrable systems in $1+1$ dimensions, by a process of dimensional and algebraic reduction. To obtain solitons moving on the line, one may either solve the reduced equations of motion [12], or else reduce wave solutions [13] of the $\mathrm{U}(n)$ Ward model along their invariance direction, say the $x$ axis. Specifying to the commutative $\mathrm{U}(2)$ case and introducing the light-cone coordinates $u=\frac{1}{2}(t+y)$ and $v=\frac{1}{2}\left(\begin{array}{ll}t & y\end{array}\right)$, I first perform a (twisted) dimensional reduction by dictating a particular $x$ dependence for the solution $\phi(u ; v ; x) 2 \mathrm{U}(2)[12]$,

$$
\phi(u ; v ; x)=\mathscr{E} \mathrm{e}^{\mathrm{i} \alpha x \sigma_{1}} g(u ; v) \mathrm{e}^{\mathrm{i} \alpha x \sigma_{1}} \mathscr{E}^{\dagger} \quad \text { for } g(u ; v) 2 \mathrm{U}(2) ;
$$

where $\mathscr{E}$ is a suitable constant 22 matrix, $\sigma_{a}$ with $a=1 ; 2 ; 3$ are the Pauli matrices, and $\alpha 2 \mathrm{R}$ is a parameter of the reduction. The Yang equation of motion (33) for $\phi$ then turns into

$$
\partial_{v}\left(g^{\dagger} \partial_{u} g\right)+\alpha^{2}\left(\sigma_{1} g^{\dagger} \sigma_{1} g \quad g^{\dagger} \sigma_{1} g \sigma_{1}\right)=0:
$$


As a second step, I algebraically restrict $g(u ; v)$ to $\mathrm{U}(1)$ via

$$
g=\mathrm{e}^{\frac{\mathrm{i}}{2} \sigma_{3} \varphi} \quad ! \quad \partial_{\nu} \partial_{u} \varphi+4 \alpha^{2} \sin \varphi=0
$$

and discover the sine-Gordon equation for the angular field $\varphi(t ; y)$.

\subsection{Time-space deformation}

For Moyal-type noncommutativity to survive the reduction to $1+1$ dimensions, I must switch from my space-space deformation to a time-space one [11],

$$
[t ; y]=\mathrm{i} \theta \quad \$ \quad[u ; v]\}=\frac{\mathrm{i}}{2} \theta ;
$$

whereupon the $g$ equation of motion (69) picks up Moyal stars,

$$
\partial_{v}\left(g^{\dagger} ? \partial_{u} g\right)+\alpha^{2}\left(\sigma_{1} g^{\dagger} ? \sigma_{1} g \quad g^{\dagger} \sigma_{1} ? g \sigma_{1}\right)=0:
$$

In this section I will stick with the star-product formulation. For the algebraic reduction, a restriction to $\mathrm{U}(1)$ is no longer appropriate, since in noncommutative $\mathrm{U}(n)$ Yang-Mills theory the overall U(1) phase no longer decouples, and one should keep its degree of freedom besides reducing $S U(2)$ to $U(1)$. With this important insight [12], my ansatz becomes

$$
g=\mathrm{e}_{?}^{\frac{\mathrm{i}}{2} \mathbb{1} \rho} ? \mathrm{e}_{?}^{\frac{\mathrm{i}}{2} \sigma_{3} \varphi} \quad 2 \quad \mathrm{U}_{?}(1) \quad \mathrm{U}_{?}(1)
$$

featuring two angular fields $\varphi$ and $\rho$. From (72) I get their equations of motion,

$$
\begin{aligned}
& \partial_{v} \mathrm{e}_{?}{ }^{\frac{\mathrm{i}}{2} \varphi} ? \partial_{u} \mathrm{e}_{?}^{\frac{\mathrm{i}}{2} \varphi}+2 \mathrm{i} \alpha^{2} \sin _{?} \varphi=\partial_{v} \mathrm{e}_{?}{ }^{\frac{\mathrm{i}}{2} \varphi} ? R ? \mathrm{e}_{?}^{\frac{\mathrm{i}}{2} \varphi} ; \\
& \partial_{v} \mathrm{e}_{?}^{\frac{\mathrm{i}}{2} \varphi} ? \partial_{u} \mathrm{e}_{?}^{\frac{\mathrm{i}}{2} \varphi} \quad 2 \mathrm{i} \alpha^{2} \sin _{?} \varphi=\partial_{v} \mathrm{e}_{?}^{\frac{\mathrm{i}}{2} \varphi} ? R ? \mathrm{e}_{?}^{\frac{\mathrm{i}}{2} \varphi ;} ;
\end{aligned}
$$

where $R=\mathrm{e}_{?} \frac{\mathrm{i}}{2} \rho$ ? $\partial_{u} \mathrm{e}_{?}^{\frac{\mathrm{i}}{2} \rho}$ carries the second scalar field $\rho$. The sum of these looses the $\alpha$ dependence and simplifies to $\partial_{v} \partial_{u} \rho=0$ in the $\theta$ ! 0 limit while the difference converges to the commutative sine-Gordon equation (70). I name (74) the "noncommutative sine-Gordon" equations [12].

\subsection{Noncommutative sine-Gordon kinks}

The equations (74) have deformed multi-kink solutions, which can be constructed via a linear system. Since from the $2+1$ perspective the waves must move in the $y$ direction, the rapidities $\mu=\mathrm{i} p 2 \mathrm{iR}$ are purely imaginary (see (35)) and, putting $x=0$, the comoving coordinate $w$ simplifies to

$$
w=\bar{\mu} u+\frac{1}{\bar{\mu}} v=\mathrm{i}\left(p u+\frac{1}{p} v\right)=\mathrm{i} \frac{y \mathrm{v} t}{\overline{1 \mathrm{v}^{2}}}=: \text { i } \eta \quad \text { with } \eta 2 \mathrm{R}:
$$

The Ward-model one-wave solution descends to the one-kink configuration [12]

$$
g=\mathscr{E}^{\dagger} \sigma_{3}(\mathbb{1} \quad 2 P) \mathscr{E} \quad \text { with projector } \quad P=T ?\left(T^{\dagger} ? T\right)_{?}^{1} ? T^{\dagger} ;
$$


where the 12 matrix function $T(\eta)$ is subject to the BPS condition

$$
\left(\partial_{\eta}+\alpha \sigma_{3}\right) T=T ? A
$$

with an arbitrary function $A(u ; v)$, which I put to zero. Conveniently fixing unimportant integration constants, the solution reads [12]

$$
\begin{aligned}
& T=\begin{array}{l}
\mathrm{e}^{\alpha \eta} \\
\mathrm{i}^{\alpha \eta}
\end{array} \quad \text { ! } \quad P=\frac{1}{2 \operatorname{ch} 2 \alpha \eta} \quad \text { i } \quad \mathrm{e}^{+2 \alpha \eta}
\end{aligned}
$$

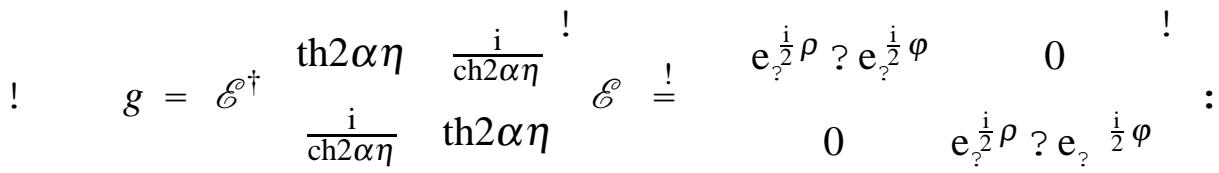

The last equation reveals that $\mathscr{E}=\begin{array}{llll}1 & 1 & 1 \\ \overline{2} & 1 & 1\end{array}$ and

$$
\mathrm{e}_{?}^{\frac{\mathrm{i}}{2} \rho} ? \mathrm{e}_{?}^{\frac{\mathrm{i}}{2} \varphi}=\operatorname{th} 2 \alpha \eta \quad \frac{\mathrm{i}}{\operatorname{ch} 2 \alpha \eta} ;
$$

which implies that $\rho=0$ and

$$
\cos _{?} \frac{\varphi}{2}=\operatorname{th} 2 \alpha \eta \quad ! \quad \tan _{?} \frac{\varphi}{4}=\mathrm{e}^{2 \alpha \eta} \quad ! \quad \varphi=4 \arctan \mathrm{e}^{2 \alpha \eta} ;
$$

which is the standard (undeformed) sine-Gordon kink with velocity $\mathrm{v}=\frac{1 p^{2}}{1+p^{2}}$. Actually, this was clear beforehand, since a soliton in $1+1$ dimensions can only depend on the single co-moving combination $\eta(u ; v)$ of the light-cone coordinates, which trivializes the star product and effectively puts $\theta=0$ and $\rho=0$. In contrast, breather and two-soliton solutions do get deformed however, according to

$$
\left[\eta_{i} ; \eta_{k}\right]_{?}=\mathrm{i} \theta\left(\begin{array}{ll}
\mathrm{v}_{i} & \mathrm{v}_{k}
\end{array}\right)^{\mathrm{P}} \overline{\left(\begin{array}{ll}
1 & \mathrm{v}_{i}^{2}
\end{array}\right)\left(\begin{array}{ll}
1 & \mathrm{v}_{k}^{2}
\end{array}\right)}:
$$

\section{SUPERSYMMETRIC EXTENSIONS}

Remembering the self-dual Yang-Mills ancestor of the Ward model, it is straightforward to supersymmetrize its noncommutative generalization [14]. The maximal $\mathscr{N}=4$ extension of noncommutative self-dual Yang-Mills reduces to a Moyal-deformed ${ }^{4} 2 \mathscr{N}=8$ super Bogomolny system $\left(F_{\alpha \beta} ; \chi_{\alpha}^{i} ; \phi^{[i j]} ; \tilde{\chi}_{\alpha}^{[i j k]} ; G_{\alpha \beta}^{[i j k l]}\right)$ subject to

$$
\begin{aligned}
& F_{\alpha \beta}+D_{\alpha \beta} H=0 \quad \text { with } D_{\alpha \beta}=\partial_{\alpha \beta}+\left[A_{\alpha \beta} ;\right] ; \\
& D_{\alpha \beta} \chi^{i \beta}+\varepsilon_{\alpha \beta}\left[H ; \chi^{i \beta}\right]=0 ; \\
& D_{\alpha \beta} D^{\alpha \beta} \phi^{i j}+2\left[H ;\left[H ; \phi^{i j}\right]\right]+2 f \chi^{i \alpha} ; \chi_{\alpha}^{j} g=0 ; \\
& D_{\alpha \beta} \tilde{\chi}^{\beta[i j k]} \quad \varepsilon_{\alpha \beta}\left[H ; \tilde{\chi}^{\beta[i j k]}\right] \quad 6\left[\chi_{\alpha}^{[i} ; \phi^{j k]}\right]=0 ; \\
& D_{\alpha}^{\gamma} G_{\gamma \beta}^{[i j k l]}+\left[H ; G_{\alpha \beta}^{[i j k l]}+12 f \chi_{\alpha}^{[i} ; \tilde{\chi}_{\beta}^{j k l]} g \quad 18\left[\phi^{[i j} ; D_{\alpha \beta} \phi^{k l]}\right] 18 \varepsilon_{\alpha \beta}\left[\phi^{[i j} ;\left[\phi^{k l]} ; H\right]\right]=0 ;\right.
\end{aligned}
$$

\footnotetext{
${ }^{4}$ As before, I only deform the bosonic spatial coordinates, and pass to the operator formulation.
} 
where $\alpha ; \beta=1 ; 2$ are $\operatorname{SO}(2,1)$ spinorial and $i ; j ; k ; l=1 ;::: ; \mathscr{N}$ are R-symmetry indices. The superspace $\mathrm{R}^{2 ; 1 \text { tै }} 3\left(x^{\alpha \beta} ; \vartheta^{i \alpha} ; \eta_{i}^{\alpha}\right)$ contains a chiral subspace $\mathrm{R}^{2 ; 1 \mathfrak{2 N}} 3$ $\left(x^{\alpha \beta} \vartheta^{i \alpha} \eta_{i}^{\beta} ; \eta_{i}^{\alpha}\right) \quad\left(y^{\alpha \beta}{ }_{i} \eta_{i}^{\alpha}\right)$, in which I introduce the Higgs superfield $\mathscr{H}$ plus chiral superfield potentials $\left(\mathscr{A}_{\alpha \beta} ; \mathscr{A}_{\alpha}^{i}\right)$ and their field strengths $\left(F_{\alpha \beta} ; \chi_{\alpha}^{i} ; \phi^{i j}\right)$ with leading component fields $\left(F_{\alpha \beta} ; \chi_{\alpha}^{i} ; \phi^{i j}\right)$. I extend the light-cone ansatz (38)-(39) to [14]

$$
\begin{aligned}
\mathscr{A}_{12} \mathscr{H} & =0 & \text { and } & \mathscr{A}_{22} & =\Phi^{1} \partial_{t+y} \Phi ; \\
\mathscr{A}_{11} & =0 & \text { and } & \mathscr{A}_{12}+\mathscr{H} & =\Phi^{1} \partial_{x} \Phi ; \\
\mathscr{A}_{1}^{i} & =0 & \text { and } & \mathscr{A}_{2}^{i} & =\Phi^{1} \partial_{2}^{i} \Phi ;
\end{aligned}
$$

and the remaining (superspace) Bogomolny equations yield equations of motion for the operator-valued $U(n)$ Yang prepotential chiral superfield $\Phi(y ; \eta)[14]$ :

$$
\begin{array}{r}
\partial_{x}\left(\Phi^{1} \partial_{x} \Phi\right)+\partial_{y t}\left(\Phi^{1} \partial_{y+t} \Phi\right)=0 ; \\
\partial_{1}^{i}\left(\Phi^{1} \partial_{x} \Phi\right)+\partial_{y t}\left(\Phi^{1} \partial_{2}^{i} \Phi\right)=0 ; \\
\partial_{1}^{i}\left(\Phi^{1} \partial_{t+y} \Phi\right) \partial_{x}\left(\Phi^{1} \partial_{2}^{i} \Phi\right)=0 ; \\
\partial_{1}^{i}\left(\Phi^{1} \partial_{2}^{j} \Phi\right)+\partial_{1}^{j}\left(\Phi^{1} \partial_{2}^{i} \Phi\right)=0 ;
\end{array}
$$

The construction of solitons then mimics the bosonic construction but takes place in the chiral superspace. The familiar co-moving coordinates get extended by a fermionic combination, $\eta_{i}=\eta_{i}^{1}+\bar{\mu} \eta_{i}^{2}$. One finds that the supersymmetric solitons are essentially a Grassmann-algebra lift of the bosonic ones. Abelian solitons, for instance, are still based on coherent states, but with parameters [14]

$$
\alpha(\eta)=\alpha^{0}+\eta_{i} \alpha^{[i]}+\eta_{i} \eta_{j} \alpha^{[i j]}+\eta_{i} \eta_{j} \eta_{k} \alpha^{[i j k]}+\eta_{i} \eta_{j} \eta_{k} \eta_{l} \alpha^{[i j k l]}:
$$

Such nilpotent additions to the soliton profiles and energy densities however do not qualitatively alter the multi-soliton dynamics [15]. A more promising option includes non-anticommutative deformations,

$$
\mathrm{f} \eta_{i}^{\alpha} ; \eta_{j}^{\beta} \mathrm{g}=C_{i j}^{\alpha \beta}
$$

replacing the Grassmann by a Clifford algebra, which should break half of the supersymmetry and might yield solitonic spin degrees of freedom.

\section{ACKNOWLEDGMENTS}

I am grateful to Abel Camacho, Claus Lämmerzahl and Alfredo Macias for having organized a wonderfully stimulating meeting. 


\section{REFERENCES}

1. R. Gopakumar, S. Minwalla and A. Strominger, JHEP 0005 (2000) 020.

2. R. Gopakumar, M. Headrick and M. Spradlin, Commun. Math. Phys. 233 (2003) 355.

3. L. Hadasz, U. Lindström, M. Roček and R. von Unge, JHEP 0106 (2001) 040.

4. M. Klawunn, O. Lechtenfeld and S. Petersen, JHEP 0606 (2006) 028.

5. O. Lechtenfeld, A.D. Popov and B. Spendig, JHEP 0106 (2001) 011.

6. O. Lechtenfeld and A.D. Popov, JHEP 0111 (2001) 040.

7. O. Lechtenfeld and A.D. Popov, Phys. Lett. B 523 (2001) 178.

8. R.S. Ward, J. Math. Phys. 29 (1988) 386; Commun. Math. Phys. 128 (1990) 319.

9. C.N. Yang, Phys. Rev. Lett. 38 (1977) 1377.

10. A.V. Domrin, O. Lechtenfeld and S. Petersen, JHEP 0503 (2005) 045.

11. C.S. Chu and O. Lechtenfeld, Phys. Lett. B 625 (2005) 145.

12. O. Lechtenfeld, L. Mazzanti, S. Penati, A.D. Popov and L. Tamassia, Nucl. Phys. B 705 (2005) 477.

13. S. Bieling, J. Phys. A 35 (2002) 6281.

14. O. Lechtenfeld and A.D. Popov, JHEP 0706 (2007) 065.

15. C. Gutschwager, T.A. Ivanova and O. Lechtenfeld, arXiv:0710.0079 [hep-th]. 